\title{
Role of microRNAs in the resistance of colorectal cancer to chemoradiotherapy (Review)
}

\author{
QI-BING WU, XIN SHENG, NING ZHANG, MING-WEI YANG and FAN WANG \\ Department of Radiotherapy, The First Affiliated Hospital of Anhui Medical University, Hefei, Anhui 230022, P.R. China
}

Received November 3, 2017; Accepted February 12, 2018

DOI: $10.3892 / \mathrm{mco} .2018 .1578$

\begin{abstract}
Colorectal cancer (CRC) is among the main tumor-related causes of death worldwide. The fact that the majority of the patients develop resistance to chemoradiotherapy (CRT) is a major obstacle for the treatment of CRC. In order to develop more effective treatment strategies, it is crucial to elucidate the mechanisms underlying the development of resistance to CRT. Several studies have recently indicated the regulatory effects of microRNAs (miRNAs) in response to antitumor agents. For example, miR-34a attenuates the chemoresistance of colon cancer to 5-FU by inhibiting E2F3 and SIRT1. The miR-34a mimic MRX34 is the first synthetic miRNA to have been entered into clinical trials. miR-21 prevents tumor cell stemness, invasion and drug resistance, which are required for the development of CRC. These findings suggest that miRNAs represent a focus in the research of novel cancer treatments aimed at sensitizing cancer cells to chemotherapeutic drugs. The aim of the present study was to review the functions of miRNAs and investigate the roles of miRNAs in CRC radioresistance or chemoresistance. Furthermore, the potential of including miRNAs in therapeutic strategies and using them as molecular biomarkers for predicting radiosensitivity and chemosensitivity was discussed.
\end{abstract}

\section{Contents}

1. Introduction

2. miRNAs and chemotherapy for CRC

3. miRNAs and radiotherapy for CRC

4. Future outlook and conclusions

Correspondence to: Professor Fan Wang, Department of Radiotherapy, The First Affiliated Hospital of Anhui Medical University, 218 Jixi Road, Shushan, Hefei, Anhui 230022, P.R. China E-mail: wangfan1965@126.com

Key words: colorectal cancer, microRNA, chemoradiotherapy resistance, biomarker

\section{Introduction}

With the improvement in living standards, changes in lifestyle and dietary structure, the incidence of colorectal cancer (CRC) has been increasing annually. According to the statistics of China's Health Care Reform in 2001, the incidence of CRC in China has risen to third among all malignant tumors. Furthermore, the mortality rate of CRC has been reported to be $10.25 / 10$ million and increasing annually. In 2015 , the incidence of CRCin China had increased from 12/10 million in the 1970 s to $56 / 10$ million, with a mean annual growth rate of $4.2 \%$, markedly exceeding the international levels.

Treatment failure is the major obstacle for the treatment of CRC (1). The chemotherapeutic agent 5-fluorouracil (5-FU) is one of the most widely applied anticancer drugs for treating CRC. Recently, a new generation of chemotherapeutic drugs, such as cetuximab (monoclonal antibody), oxaliplatin (a drug widely used in the treatment of CRC) and panitumumab (a targeted biological agent), have been applied in clinical practice $(2,3)$. However, acquired and inherent resistance to treatment represent a major challenge in the management of CRC. Another common treatment for the CRC is radiation therapy. However, the efficacy of radiation therapy depends on the radiosensitivity of the tumor. In addition, the extent of response to radiation therapy varies among different tissues, organs and types of tumors, due to different degrees of radiosensitivity or radioresistance. To predictthe sensitivity or resistance of CRC patients to specific chemotherapeutic drugs and radiotherapy, the mechanisms underlying sensitivity or resistance to treatment must be elucidated.

It has been demonstrated that microRNAs (miRNAs) play avital role in the regulation of genes exerting antitumor effects, such as genes driving cell apoptosis, inhibiting cell proliferation and regulating drug efflux mechanisms (4-7). miRNAs are a class of small, endogenous, non-coding, regulatory RNA molecules. miRNAs regulate the expression of their target genesthrough complementing the 3'-untranslated region of the target genes leading to target mRNA cleavage or translation inhibition. Earlier studies demonstrated that miRNAs are associated with the regulation of cell differentiation, proliferation and death inbasic biological processes (8-10). miRNA-mediated gene regulation is an important process in the occurrence, pathogenesis and progression of several diseases $(11,12)$. It has been demonstrated that miRNAs are abnormally expressed in a variety of human diseases, 
such as autoimmune, cardiovascular, cerebrovascular, neurodegenerative and metabolic diseases, as well as cancer $(6,13)$. As regards tumor occurrence and development, miRNAs may act as oncogenes or tumor suppressors. miRNAs may act as oncogenes, referred to as 'oncomirs', through inhibiting the effect of tumor suppressor genes or regulating cell apoptosis, promoting the occurrence and development of tumors $(11,14,15)$. The identification of miRNAs implicated in the response to antitumor therapy has been shown to promote the generation of new therapeutic approaches to the reversal of drug resistance (16). However, there remain several issues to be addressed: i) Although the mechanisms through which miRNAs induce oncogenesis and tumor progression have been previously investigated and largely clarified, the role of miRNAs requires further elucidation. ii) The role of miRNAs in cancer chemotherapy and radiotherapy remains ambiguous. iii) The mechanism of action and role of miRNAs in the development of resistance to chemoradiotherapy (CRT) are complex. iv) The expression profiles of miRNAs in different tumors have not been fully established. v) The use of miRNAs as biomarkers also require further investigation. Therefore, it is crucial to understand the mechanism of action of miRNAs in regulating tumor resistance to radiotherapy or chemotherapy through regulating biological processes and signaling pathways.

\section{2. miRNAs and chemotherapy for CRC}

Chemotherapy for CRC. Chemotherapy is an important treatment strategy for solid tumors, and one of the main treatment methods for CRC $(17,18)$. Apoptosis is one of the main signs of effective anticancer chemotherapy; however, due to drug resistance, the apoptotic effects of drugs on the tumor cells are weakened and a large proportion of patients respond poorly to chemotherapy $(19,20)$. At present, drug resistance of tumor cells is one of the main reasons for chemotherapy failure (21). Therefore, it is of great clinical significance to elucidate the mechanism underlying the development of drug resistance of tumor cells, in order to reverse this process and improve the sensitivity of tumor cells to chemotherapeutic agents (22). 5-FU, the most common apoptosis-inducing agent among chemotherapeutic drugs, mainly induces apoptosis through enhancing or reducing the levels of B-cell lymphoma (Bcl)-2 family proteins. Resistance to 5-FU represents a major challenge incancer treatment (23).

miRNA treatment for CRC. In recent years, research has focused on the role of miRNAs in the chemotherapy of CRC. Zhou et al (22) reported that the inhibition of miR-21 and miR-200b expression may improve the sensitivity of cholangiocarcinoma cells to gemcitabine. Borralho et al observed that miR-143 expression in the human CRC cell line HCT116 significantly increased the apoptosis of colon cancer cells exposed to 5-FU by inhibiting expression of extracellular-regulated protein kinase 5 (ERK5), nuclear factor $\kappa \mathrm{B}(\mathrm{NF}-\kappa \mathrm{B})$ and Bcl-2 (24,25). Therefore, overexpression of miR-143 leads to reduction of ERK5 and NF- $\mathrm{KB}$ signaling, both of which may increase the chemosensitivity of CRC cells (25).

miRNA-192 and miRNA-215 may trigger the ability of CRC cells to resist5-FU treatment, and mRNA-215 may reduce the chemosensitivity of $\mathrm{CRC}$ cells to methotrexate and raltitrexed (26). Several studies have demonstrated that overexpression of miR-10b may promoteresistance to chemotherapy in vitro by targeting the pro-apoptotic factor Bcl-2-like protein 11 (BIM). The functions of BIM are mediated by activating Bax or inhibiting Bcl-2-mediated apoptosis $(24,27)$. miRNA-10b overexpression has been associated with poor prognosis (28). Overexpression of the tumor suppressor miR-96 may induce cell apoptosis and increase the sensitivity of human CRC cells to 5-FU. miRNA-96 regulates the expression ofthe anti-apoptosis factorsX-linked inhibitor of apoptosis protein and ubiquitin-conjugating enzyme E2 Nby indirectly targeting these two proteins $(29,30)$. Therefore, inhibition of this gene can enhance apoptosis and restore the sensitivity of cancer cells to 5-FU.

Previous studies demonstrated that miRNA-587 may enhance resistance to $5-\mathrm{FU}$ by reducing serine/threonine protein kinase 2A regulatory subunit 1B (PPP2R1B) $(17,18)$. By contrast, inhibition of miRNA-587 may enhance the expression of PPP2R1B in CRC cells, which increases sensitivity to 5-FU treatment. Previous studies indicated that miRNA-302a expression is decreased in CRC cells, and high expression of miRNA-302a inhibits cell proliferation through inactivating ERK1/2 and PI3K/AKT $(17,20)$. Oxaliplatin (L-OHP) is a third-generation platinum-based chemotherapy agent. The combination of L-OHP and 5-FU has been registeredas a standard adjuvant chemotherapy for stage III CRC. A previous study demonstrated that a total of 56 miRNAs are upregulated and 50 are downregulated in cells exposed to 5-FU, or the L-OHP-sensitive colon cancer cells HCT-8 and HCT-116 (2).

Combining treatment with miRNAs and chemotherapy for CRC. A number of miRNAs may be involved in chemotherapy resistance and poor prognosis in colon cancer. Qian et al (31) reported that IGF-1R is a direct target gene of miR-143, and the expression of miR-143 can improve the sensitivity of CRC cells to L-OHP. In clinical studies, Hansen et al (32) enrolled 89 patients with advanced CRC who received first-line treatment with $\geq 3$ cycles of XELOX. Subsequently, the miR-126 level was measured using in situ hybridization in primary tumor tissue. The results revealed that the effect of miR-126 was associated with the XELOX treatment success rate; patients with a high level of miR-126 expression had a mean survival time of 11.5 months, whereas patients with a low level of miR-126 expression had a survival time of6 months (32).

CPT-11 is a camptothecin derivative that is mainly used in the treatment of advanced CRC. Tong et al (33) used a miRNA chip to detect miRNA expression in the SW1116 and SW1116/HCPT cell lines (hydroxycamptothecin-resistant), and found 77 differentially expressed miRNAs. They observed that miR-506 attenuates the expression of PPAR $\alpha$, which mediates drug resistance tohydroxycamptothecin. The ATP binding cassette transporter $\mathrm{G} 2$ (ABCG 2) gene is a member of the family of $\mathrm{ABC}$ transporters. ABCG 2 overexpression can increase resistance of cancer cells to chemotherapeutic agents. It was recently demonstrated that single-nucleotide polymorphisms(SNPs) in miRNA biosynthetic genes and miRNA genomic regions may affect the development and treatment of tumors (33). Boni et al (34) studied 61 rectal cancer patients with metastatic lymph nodes undergoing 5-FU and CPT-11 treatment, and discovered a significant correlation between disease-free survival and the TT genotype 
of the pri-miR216a-1 gene. In addition, SNP rs1834306 of pri-miR-100 was associated with longer disease progression time. That study was the first to suggest an association between the treatment results of the miRNA biosynthetic pathway and the miRNA SNP rs11077. Furthermore, they also indicated that the disease is associated with the nucleocytoplasmic transport protein exportin-5. Thus, these miRNAs and their targeting genes may serve as new molecular markerstopredict the response to chemotherapy and design novel targeted therapies.

\section{3. miRNAs and radiotherapy for CRC}

Preoperative radiotherapy has become a standard method for the treatment of patients with locally advanced CRC. miRNAs are promising biomarkers for predicting personalized responses. A recent study demonstrated that miR-198, miR-765, miR-630, miR-371-5p, miR-575, miR-202 and miR-513a-5p maybe used for predicting the response of CRC to preoperative radiotherapy (35). In addition, miR-630 expression is positively correlated with radiosensitivity in CRC cell lines. Post-radiotherapy, miR-630 induces CRC cell apoptosis and causes a reduction of CRC cell resistance to radiotherapy. Moreover, the action of miR-630 was found to be dependent on BCL2L2 and TP53RK, both of which are target genes of miR-630 and can induce cancer cell resistance to radiotherapy. TP53RK is an atypical protein kinase that was first identified in an interleukin-2-activated cytotoxic T-cell subtraction library and has been shown to bind and phosphorylate p53 in the presence of an activating cellular lysate (36). Therefore, miR-630 induces cancer cell resistance to radiotherapy by triggering TP53RK. In addition, it was also demonstrated that CREB regulates the expression of miR-630 (36). Following X-ray irradiation, miR-100 was obviously downregulated in CCL-244 cells, indicating that miR-100 may play an important role in regulating $\mathrm{CRC}$ radiosensitivity, and it may act as a new clinical target for CRC radiotherapy (37). Radiotherapy may induce miR-622 overexpression in colon cancer cells; conversely, miR-622 causes radioresistance and poor response to further therapy. miR-622 is a potential molecular marker of responders to radiotherapy, as well as a potential therapeutic target (38). A previous study reported that miR-106b may increase CRC cell radioresistance by directly inhibiting PTEN and p21 (39). Yin et al (40) demonstrated that $\geq 4$ gene expression changes occurred in the brain of irradiated mice. As miRNAs may regulate multiple genes simultaneously, the results indicate that the miRNA regulation and the subsequent regulation of a large number of target genes may occur quickly within a short time period. Recent studies have suggested that miRNAs are important participants in the cellular stress response induced by radiation therapy. Zhang et al (41) observed that miR-124 directly inhibits the PRRX1 protein, which renders human CRC cells more sensitive to ionizing radiation in vivo or in vitro. The PRRX1 protein is a major regulator of epithelial-to-mesenchymal transition, suggesting that miR-124 maybe used as a potential biomarker for early prediction of CRC and prognosis (41).

Another study demonstrated that miR-62 overexpression may confer radioresistanceby activating the apoptosis gene. miR-622 is a potential biomarker and a potential therapeutic target for patients who do not respond to radiation therapy (38).
Another important regulatory factor in CRC is miR-100. It has been demonstrated that the upregulation of miR-100 significantly increases the expression of X-linked inhibitor of apoptosis and other apoptosis-related proteins in CLL-244 cells. One of the most important mechanisms involved in radiation-induced cell damage is the induction of DNA double-strand breaks (DSBs). Yang et al (37) demonstrated that miR-100 regulates the sensitivity of CLL-244 cells to radiation by increasing radiation-induced DNA DSBs.Therefore, miRNAs contribute to the radioresistance or chemoresistance of CRC. Moreover, although a number ofmiRNAs have been found to be involved in the regulation of radioresistance or chemoresistance, the specific underlying mechanisms have not been fully elucidated, which constitutesa limitation of the present study.

\section{Conclusions and future perspectives}

Although several miRNAs have been implicated in the response to chemotherapy or radiotherapy, we have not yet identified a miRNA that is involved in both chemo-and radio-sensitivity, or a miRNA that affects both chemo- and radio-sensitivity in the same patient. Future studies should focus on miRNAs involved in both chemo-and radiosensitivity. Not all chemotherapy- and radiotherapy-associated miRNAs have been included in the present study; other miRNAs associated with the response to CRT will be investigated in future studies. Drug resistance remains a major challenge in clinical treatment, and is also one of the main causes of recurrent CRC. It is crucial to select the appropriate treatment for each patient, as suboptimal treatment will lead to cancer progression and a dismal outcome. The role of miRNA is to regulate targeted mRNAs and/or proteins. An increasing number of experts pointed out that miRNAs maybe used to predict and determine the response to conventional anticancer therapies, and can be used to prevent exposure of non-responders to ineffective therapy. miRNAs may change current treatment strategies to make them more targeted and effective. Accumulating evidence demonstrates that the response of miRNAs directly affect cancer treatment. A strong basis for miRNA use is to overcome the resistance of cancer cells to chemotherapy, radiotherapy and targeted therapy, and restoring drug sensitivity. miRNAs may be used as an effective therapeutic method for targeted treatment to enhance tumor response oras predictive markers for treatment outcomes. Within the next 5 years, miRNAs may also be used for the development of novel drugs. There is a specific trend for miRNAs to be used as biomarkers for prediction of chemotherapy resistance, which may guide patient selection and optimize treatment with minimal toxicity. An increasing number of miRNAs are to be investigated in the immediate future, and the role of those miRNAs in the resistance to CRT will be more clearly delineated, which will hopefully promote the development of new strategies for cancer treatment. New drugs acting on miRNAs may resolve several obstacles in cancer treatment. The main aim is to identify miRNAs as therapeutic targets in personalized therapy and to identify patients who may benefit from such treatment. Recent advances in the development of miRNA-based anticancer therapies have produced promising results. A more profound understanding of the mechanisms underlying the involvement of miRNAs 
in anticancer drug resistance will lead to the development of more effective treatment methods.

\section{Acknowledgements}

Not applicable.

\section{Funding}

This study was supported by the China International Medical Foundation of Science anti-tumor therapy special scientific research (grant no. Z-2014-06-16355).

\section{Availability of data and materials}

The analysed data sets generated during the study are available from the corresponding author on reasonable request.

\section{Authors' contributions}

Q-BW and FW designed study and wrote the manuscript. Q-BW, XS, NZ and M-WY performed literatures search regarding miRNAs and cancer therapy. FW was responsible for acquisition of funding for the present study. All authors critically revised the manuscript.

\section{Ethics approval and consent to participate}

Not applicable.

\section{Consent for publication}

Not applicable.

\section{Competing interests}

The authors declare that they have no competing interests.

\section{References}

1. Tauriello DV, Calon A, Lonardo E and Batlle E: Determinants of metastatic competency in colorectal cancer. Mol Oncol 11: 97-119, 2017.

2. Kurkjian C and Kummar S: Advances in the treatment of metastatic colorectal cancer. Am J Ther 16: 412-420, 2009.

3. Nie P, Hu W, Zhang T, Yang Y, Hou B and Zou Z: Synergistic Induction of Erlotinib-Mediated Apoptosis by Resveratrol in Human Non-Small-Cell Lung Cancer Cells by Down-Regulating Survivin and Up-Regulating PUMA. Cell Physiol Biochem 35: 2255-2271, 2015.

4. Yu HW and Cho WC: The emerging role of miRNAs in combined cancer therapy. Expert Opin Biol Ther 15: 923-925, 2015.

5. Zhao Y and Srivastava D: A developmental view of microRNA function. Trends Biochem Sci 32: 189-197, 2007.

6. Ao X, Nie P, Wu B, Xu W, Zhang T, Wang S, Chang H and Zou Z: Decreased expression of microRNA-17 and microRNA-20b promotes breast cancer resistance to taxol therapy by upregulation of NCOA3. Cell Death Dis 7: e2463, 2016.

7. Zou Z, Luo X, Nie P, Wu B, Zhang T, Wei Y, Wang W, Geng G, Jiang J and Mi Y: Inhibition of SRC-3 enhances sensitivity of human cancer cells to histone deacetylase inhibitors. Biochem Biophys Res Commun 478: 227-233, 2016.

8. Amirkhah R, Schmitz U, Linnebacher M, Wolkenhauer O and Farazmand A: MicroRNA-mRNA interactions in colorectal cancer and their role in tumor progression. Genes Chromosomes Cancer 54: 129-141, 2015
9. Fiore R, Siegel G and Schratt G: MicroRNA function in neuronal development, plasticity and disease. Biochim Biophys Acta 1779: 471-478, 2008.

10. Xu DR, Huang S, Long ZJ, Chen JJ, Zou ZZ, Li J, Lin DJ and Liu Q: Inhibition of mitotic kinase Aurora suppresses Akt-1 activation and induces apoptotic cell death in all-trans retinoid acid-resistant acute promyelocytic leukemia cells. J Transl Med 9: 74, 2011.

11. Jiang Q, Wang Y, Hao Y, Juan L, Teng M, Zhang X, Li M, Wang $G$ and Liu Y: miR2Disease: A manually curated database for microRNA deregulation in human disease. Nucleic Acids Res 37 (Database): D98-D104, 2009.

12. Gao J, Zou Z, Gao J, Zhang H, Lin Z, Zhang Y, Luo X, Liu C, Xie J and Cai C: Increased expression of HMGB3: A novel independent prognostic marker of worse outcome in patients with esophageal squamous cell carcinoma. Int J Clin Exp Pathol 8: 345-352, 2015.

13. Zhu J, Zou Z, Nie P, Kou X, Wu B, Wang S, Song Z and He J: Downregulation of microRNA-27b-3p enhances tamoxifen resistance in breast cancer by increasing NR5A2 and CREB1 expression. Cell Death Dis 7: e2454, 2016.

14. Calin GA and Croce CM: MicroRNA signatures in human cancers. Nat Rev Cancer 6: 857-866, 2006.

15. Luo X, Yao J, Nie P, Yang Z, Feng H, Chen P, Shi X and Zou Z: FOXM1 promotes invasion and migration of colorectal cancer cells partially dependent on HSPA5 transactivation. Oncotarget 7: 26480-26495, 2016.

16. Mi YJ, Geng GJ, Zou ZZ, Gao J, Luo XY, Liu Y, Li N, Li CL, Chen YQ, Yu XY, et al: Dihydroartemisinin inhibits glucose uptake and cooperates with glycolysis inhibitor to induce apoptosis in non-small cell lung carcinoma cells. PLoS One 10: e0120426, 2015.

17. Chen P, Luo X, Nie P, Wu B, Xu W, Shi X, Chang H, Li B, Yu X and Zou Z: CQ synergistically sensitizes human colorectal cancer cells to SN-38/CPT-11 through lysosomal and mitochondrial apoptotic pathway via p53-ROS cross-talk. Free Radic Biol Med 104: 280-297, 2017.

18. Lou YF, Zou ZZ, Chen PJ, Huang GB, Li B, Zheng DQ, Yu XR and Luo XY: Combination of gefitinib and DNA methylation inhibitor decitabine exerts synergistic anti-cancer activity in colon cancer cells. PLoS One 9: e97719, 2014.

19. Nita ME, Nagawa H, Tominaga O, Tsuno N, Fujii S, Sasaki S, Fu CG, Takenoue T, Tsuruo T and Muto T: 5-Fluorouracil induces apoptosis in human colon cancer cell lines with modulation of Bcl-2 family proteins. Br J Cancer 78: 986-992, 1998.

20. Zou Z-Z, Nie P-P, Li Y-W, Hou B-X, Li R, Shi X-P, Ma Z-K, Han B-W and Luo X-Y: Synergistic induction of apoptosis by salinomycin and gefitinib through lysosomal and mitochondrial dependent pathway overcomes gefitinib resistance in colorectal cancer. Oncotarget 8: 22414-22432, 2015.

21. Zou Z, Yuan Z, Zhang Q, Long Z, Chen J, Tang Z, Zhu Y, Chen S, $\mathrm{Xu}$ J, Yan M, et al: Aurora kinase A inhibition-induced autophagy triggers drug resistance in breast cancer cells. Autophagy 8 : 1798-1810, 2012.

22. Zhou W, Xu J, Gelston E, Wu X, Zou Z, Wang B, Zeng Y, Wang H, Liu A, Xu L, et al: Inhibition of Bcl-xL overcomes polyploidy resistance and leads to apoptotic cell death in acute myeloid leukemia cells. Oncotarget 6: 21557-21571, 2015.

23. Zhang H, Tang J, Li C, Kong J, Wang J, Wu Y, Xu E and Lai M: miR-22 regulates 5-FU sensitivity by inhibiting autophagy and promoting apoptosis in colorectal cancer cells. Cancer Lett 356 (2 Pt B): 781-790, 2015.

24. Czabotar PE, Colman PM and Huang DC: Bax activation by Bim? Cell Death Differ 16: 1187-1191, 2009.

25. Borralho PM, Kren BT, Castro RE, da Silva IB, Steer CJ and Rodrigues CM: MicroRNA-143 reduces viability and increases sensitivity to 5-fluorouracil in HCT116 human colorectal cancer cells. FEBS J 276: 6689-6700, 2009.

26. Wu S, Fesler A and Ju J: Implications of Circadian Rhythm Regulation by microRNAs in Colorectal Cancer. Cancer Transl Med 2: 1-6, 2016.

27. Marani M, Tenev T, Hancock D, Downward J and Lemoine NR: Identification of novel isoforms of the $\mathrm{BH} 3$ domain protein Bim which directly activate Bax to trigger apoptosis. Mol Cell Biol 22: 3577-3589, 2002.

28. Nishida N, Yamashita S, Mimori K, Sudo T, Tanaka F, Shibata K, Yamamoto H, Ishii H, Doki Y and Mori M: MicroRNA-10b is a prognostic indicator in colorectal cancer and confers resistance to the chemotherapeutic agent 5-fluorouracil in colorectal cancer cells. Ann Surg Oncol 19: 3065-3071, 2012. 
29. Kim SA, Kim I, Yoon SK, Lee EK and Kuh HJ: Indirect modulation of sensitivity to 5-fluorouracil by microRNA-96 in human colorectal cancer cells. Arch Pharm Res 38: 239-248, 2015.

30. Zhou J, Zhou Y, Yin B, Hao W, Zhao L, Ju W and Bai C: 5-Fluorouracil and oxaliplatin modify the expression profiles of microRNAs in human colon cancer cells in vitro. Oncol Rep 23: 121-128, 2010.

31. Qian X, Yu J, Yin Y, He J, Wang L, Li Q, Zhang LQ, Li CY, Shi ZM, Xu Q, et al: MicroRNA-143 inhibits tumor growth and angiogenesis and sensitizes chemosensitivity to oxaliplatin in colorectal cancers. Cell Cycle 12: 1385-1394, 2013.

32. Hansen TF, Sørensen FB, Lindebjerg J and Jakobsen A: The predictive value of microRNA-126 in relation to first line treatment with capecitabine and oxaliplatin in patients with metastatic colorectal cancer. BMC Cancer 12: 83, 2012.

33. Tong JL, Zhang CP, Nie F, Xu XT, Zhu MM, Xiao SD and Ran ZH: MicroRNA 506 regulates expression of PPAR alpha in hydroxycamptothecin-resistant human colon cancer cells. FEBS Lett 585: 3560-3568, 2011.

34. Boni V, Zarate R, Villa JC, Bandrés E, Gomez MA, Maiello E, Garcia-Foncillas J and Aranda E: Role of primary miRNA polymorphic variants in metastatic colon cancer patients treated with 5-fluorouracil and irinotecan. Pharmacogenomics J 11: 429-436, 2011.

35. Zhu Y, Peng Q, Lin Y, Zou L, Shen P, Chen F, Min M, Shen L, Chen J and Shen B: Identification of biomarker microRNAs for predicting the response of colorectal cancer to neoadjuvant chemoradiotherapy based on microRNA regulatory network. Oncotarget 8: 2233-2248, 2017.
36. Zhang Y, Yu J, Liu H, Ma W, Yan L, Wang J and Li G: Novel Epigenetic CREB-miR-630 Signaling Axis Regulates Radiosensitivity in Colorectal Cancer. PLoS One 10: e0133870, 2015.

37. Yang XD, Xu XH, Zhang SY, Wu Y, Xing CG, Ru G, Xu HT and Cao JP: Role of miR-100 in the radioresistance of colorectal cancer cells. Am J Cancer Res 5: 545-559, 2015.

38. Ma W, Yu J, Qi X, Liang L, Zhang Y, Ding Y, Lin X, Li G and Ding Y: Radiation-induced microRNA-622 causes radioresistance in colorectal cancer cells by down-regulating $\mathrm{Rb}$. Oncotarget 6: 15984-15994, 2015.

39. Zheng L, Zhang Y, Liu Y, Zhou M, Lu Y, Yuan L, Zhang C, Hong M, Wang S and Li X: MiR-106b induces cell radioresistance via the PTEN/PI3K/AKT pathways and p21 in colorectal cancer. J Transl Med 13: 252, 2015

40. Yin E, Nelson DO, Coleman MA, Peterson LE and Wyrobek AJ: Gene expression changes in mouse brain after exposure to low-dose ionizing radiation.Int J Radiat Biol 79:759-775, 2003.

41. Zhang Y, Zheng L, Huang J, Gao F, Lin X, He L, Li D, Li Z, Ding Y and Chen L: MiR-124 Radiosensitizes human colorectal cancer cells by targeting PRRX1. PLoS One 9: e93917, 2014. 